\title{
LA INTERVENCIÓN EN TRABAJO SOCIAL DESDE LA CALIDAD INTEGRADA
}

\section{CARMEN BARRANCO EXPÓSITO}

Trabajadora Social. Doctora en Psicología. Profesora Titular de Trabajo Social. Departamento de Trabajo Social y Servicios Sociales. Centro Superior de Ciencias Políticas y Sociales de la Universidad de La Laguna.

"La incorporación de la calidad es una exigencia ética para los sistemas de bienestar social (...) la finalidad perseguida no debe ser la mejora de la calidad de los servicios sino la mejora de la calidad de vida de las personas".

BARRIGA, 2000

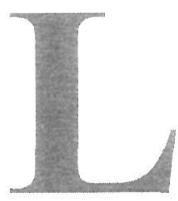

\section{RESUMEN}

a intervención en el Trabajo Social es entendida como la acción organizada y desarrollada por los trabajadores sociales con las personas, grupos y comunidades. Sus objetivos están orientados a superar los obstáculos que impiden avanzar en el desarrollo humano y en la mejora de la calidad de vida de la ciudadanía. Intervención profesional que se basa en los fundamentos éticos, epistemológicos y metodológicos del Trabajo Social, desde un enfoque global, plural y de calidad.

Estos planteamientos del Trabajo Social parten de una concepción dinámica de la persona que se inscribe en un contexto social, histórico, político, económico y cultural de la realidad social, conectando con otras disciplinas y profesionales. Estos son algunos de los aspectos que se abordan en este artículo y, concretamente, se plantea responder a las siguientes cuestiones: ¿qué es y cuáles son las características de la intervención en Trabajo Social? y ¿para qué intervenir en Trabajo Social desde la calidad integrada en las organizaciones de servicios sociales y de bienestar social?

\section{ABSTRACT}

Intervention in Social Work is intended here as the social workers' organized action with people, groups and communities. Their objectives 
are oriented to overcome obstacles impeding the human development and to enhance citizens' quality of life. This professional intervention is based upon the ethical, episthemological and methodological foundations of the Social Work from a global, plural and quality leaded focus.

This point of view, Social Work depart from a dinamic conception of person embedded in a social, historical, political, economic, and cultural context of social reality, connecting with other disciplines and professionals. These are some of the aspects approached in this paper and, specifically, we try to answer the following questions: what and which are the characteristics of Social Work intervention? and Why should we intervene in Social Work from a integrated quality point of view within social services and welfare organizations?

\section{I. ¿QUÉ ES Y CUÁLES SON LAS CARACTERÍSTICAS DE LA INTERVENCIÓN EN TRABAJO SOCIAL?}

Etimológicamente, el término "intervención" hace referencia a la acción y efecto de intervenir; la palabra "intervenir" significa tomar parte en un asunto y también interceder o mediar por alguien (Diccionario de la Real Academia Española, 2001).

Aplicado al Trabajo Social, la intervención es la acción organizada y desarrollada por los trabajadores sociales con las personas, grupos y comunidades. Para la trabajadora social y profesora María Asunción Martínez Román (2003), dicha intervención está orientada a acompañar, ayudar y capacitar a las personas en sus procesos vitales para ser responsables, para ser libres de elegir y ejercer la participación, así como a facilitar los cambios de aquellas situaciones que supongan un obstáculo para el desarrollo humano y la justicia social, promoviendo los recursos de la política social, las respuestas innovadoras y la creación de recursos no convencionales, basados en el potencial solidario de las personas. Para tal fin, afirma la autora que no sólo se actúa profesionalmente con la gente y su ambiente más próximo, como es la familia, amigos y vecinos, sino que se intenta intervenir también en los contextos que condicionan o limitan sus posibilidades de desarrollo, teniendo como referentes la Declaración Internacional de los Derechos Humanos y la promoción de la justicia.

De ahí que la intervención en Trabajo Social esté centrada tanto en la persona como en el medio social, partiendo de un enfoque global y plural. Intervención profesional que se inscribe en un contexto social, histórico, político, económico y cultural de la realidad social. La misma 
parte de los fundamentos éticos, epistemológicos y metodológicos del Trabajo Social, basados en una concepción dinámica de la persona y del mundo. Los objetivos específicos y los modos concretos de llevar a cabo la intervención están supeditados a las características de la realidad de las personas y del contexto social, a los niveles y modelos de intervención del Trabajo Social desde los cuales se intervenga.

A partir de estas consideraciones previas sobre la disciplina y la profesión, vamos a señalar algunas de las características generales de la intervención en Trabajo Social, las cuales se representan en el siguiente dibujo.

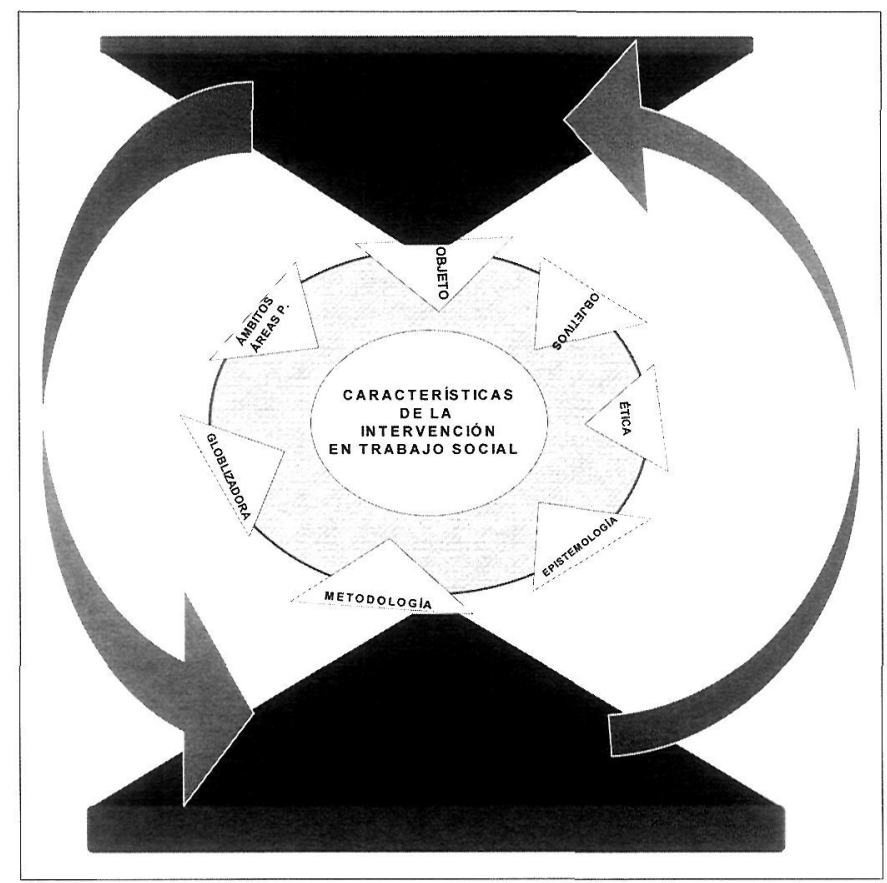

Fuente: elaboración propia.

Entre las principales características, observamos las referidas al objeto, los objetivos, la ética profesional, la epistemología, la metodología, la función globalizadora, los ámbitos de intervención y las áreas profesionales de actuación, que pasamos a esbozar muy brevemente.

\subsection{Objeto del Trabajo Social}

La intervención profesional de los trabajadores sociales parte del objeto de la disciplina, que está centrado en las situaciones de dificultad de las personas y los obstáculos del medio social que limitan o impiden 
el desarrollo humano. Situaciones que precisan de la intervención del trabajador social para transformar, mejorar o superar las dificultades, potenciar las capacidades y recursos, incidiendo tanto en las situaciones individuales, grupales o comunitarias, como en las del medio social.

\subsection{Objetivos generales del Trabajo Social.}

Los mismos tratan de generar cambios para ayudar a las personas, grupos o comunidades, a satisfacer sus necesidades; a superar las dificultades materiales y no materiales, los problemas sociales y los obstáculos que impiden o limitan la igualdad de oportunidades; a potenciar las capacidades de las personas; así como a contribuir a promover el bienestar social, el desarrollo humano y la calidad de vida de la ciudadanía. El tipo de cambio estará supeditado a las situaciones de las personas y a las condiciones del medio social, así como a los propios enfoques de los modelos de intervención en el que se inscriba la acción profesional, pudiendo dar lugar a una transformación, modificación o mejora.

\subsection{Etica del Trabajo Social.}

La intervención social parte de los valores filosóficos y los principios éticos de los Derechos Humanos de la dignidad a la persona, respeto, autodeterminación, justicia social, igualdad de oportunidades y participación recogidos en el Código de Ética Internacional del Trabajo Social (Consejo General de Colegios Oficiales de Diplomados en Trabajo Social y Asistentes Sociales, 1999). Igualmente, se especifica la obligación que tienen los trabajadores sociales con la disciplina y con la sociedad. Vinculado con la calidad, se especifica el deber de proporcionar la mejor atención a todas aquellas personas que soliciten su ayuda y asesoramiento; motivar a los clientes a que participen lo más posible, así como la responsabilidad profesional con las organizaciones, la sociedad y otros profesionales.

\subsection{La Epistemología en Trabajo Social.}

Desde la pluralidad paradigmática que caracteriza a las Ciencias Sociales, en las que se incluye nuestra disciplina, el Trabajo Social comparte la diversidad de perspectivas paradigmáticas de las cuales emanan los distintos modelos de intervención que han ido elaborando, deconstruyendo y reconstruyendo los trabajadores sociales.

En esta dirección, se afirma que, desde los paradigmas de las Ciencias Sociales, el Trabajo Social se fundamenta en los modelos interaccionistas centrados en la persona y en la situación, con un enfoque global que enriquece las recíprocas interacciones, entre la persona 
y el medio social (Red de la, 1996). Modelos de intervención profesional que, a través de las diversas perspectivas paradigmáticas, focalizan la intervención de distinta manera. Así, los modelos correspondientes a la perspectiva psicodinámica, psicosocial y los de la perspectiva conductista-cognitiva están más encaminados a la capacitación de las personas para que éstas afronten sus dificultades y avancen en su propio desarrollo personal y social.

Por otra parte, los modelos críticos están más orientados a promover cambios en la sociedad y en las propias personas, el empoderamiento y la defensa de sus derechos sociales, a través de los procesos dialécticos, participativos y colectivos. En estos últimos modelos priman el nivel colectivo para encarar los propios cambios personales y la transformación de las estructuras sociales generadoras de exclusión y desigualdad social, con la finalidad de avanzar hacia el desarrollo humano, la calidad de vida y la justicia social. Y por último, los modelos desde la perspectiva sistémica-ecológica, que se orientan más hacia la intervención con las personas, las redes sociales y la naturaleza. Su finalidad es la de potenciar los procesos de capacitación personal y la calidad de vida, así como el desarrollo social y un medio ambiente ecológico y sostenible.

También, conviene señalar que en los últimos años se resalta la necesidad de la intervención multinivel. Este enfoque preconiza una práctica profesional integradora que una el trabajo individual, familiar, grupal y comunitario. Además, que integre diferentes teorías y métodos, lo micro y macro, así como lo objetivo y lo subjetivo (Red de la, 2000; Reid, 2003).

\subsection{La metodología en Trabajo Social.}

Actualmente en Trabajo Social, a nivel individual, grupal y comunitario, se aplica el método o los métodos de las Ciencias Sociales, según se parta de una concepción unitaria del método o del pluralismo metodológico. En cualquier caso, el método o los métodos en Trabajo Social presentan una estructura y procesos que se configuran en torno a las fases interrelacionadas que posibilitan conocer, diagnosticar, establecer un plan de acción, ejecutar dicho plan y evaluar los procesos y los resultados alcanzados. De esta manera, se logra profundizar en el conocimiento e intervenir con las personas, grupos o comunidades para producir cambios o transformar la realidad social. Lógicamente, las técnicas y el modo de implementar las fases y procesos están supeditados a los paradigmas de estudio o investigación que se apliquen, así como los modelos y niveles de intervención. 


\subsection{Perspectiva Globalizadora.}

La intervención profesional se realiza desde una perspectiva globalizadora, intradisciplinar e interdisciplinar, para profundizar y dar una respuesta más efectiva a las situaciones complejas que caracterizan la realidad social. Para tal fin, los trabajadores sociales comparten ámbitos de intervención con otros profesionales y se coordinan con psicólogos, pedagogos, sociólogos, abogados, médicos, etc. También están en conexión con los agentes políticos, sindicales y sociales de la acción social.

Por otra parte, cabe resaltar que se está fomentando la cooperación entre organizaciones y el tejido asociativo y sector económico para abordar lo que se viene denominando el "trabajo en red". A través de estas redes se establece la implicación de los entes públicos y privados, vertebrados en torno a programas que van desde lo local a lo transnacional. Se trata de dar una repuesta global e integral, incluyendo las áreas y sectores, abarcando tanto la planificación, ejecución, evaluación y coordinación de las acciones para obtener resultados eficaces en los diversos ámbitos de intervención profesional.

Esta perspectiva global y aplicada al medio rural, Rueda (2000), expresa que la intervención se ha de abordar desde los principios básicos de multidimensionalidad, cooperación, coordinación, interdisciplinariedad y transversalidad. A los que, en mi opinión, habría que añadir el de la calidad integrada.

\section{7. Ámbitos de intervención y áreas profesionales.}

La intervención en Trabajo Social se realiza en los diversos ámbitos de los Servicios Sociales y de Bienestar Social, así como en otros ámbitos comunitarios, el sociosanitario, de Derechos Humanos, etc. La misma se lleva a cabo en las distintas Administraciones Públicas (Central, Autonómica y Local), en las ONGs y en el sector mercantil.

En estos ámbitos de intervención profesional, los trabajadores sociales realizan diversas actividades que se agrupan en las denominadas "áreas profesionales", siendo definida el área profesional como: "la agrupación de funciones profesionales relacionadas" (Consejo General de Colegios Oficiales de Diplomados en Trabajo Social y Asistentes Sociales de España, 2003, 17). A su vez, el área se define como "la capacidad de acción propia de los cargos y oficios". (Diccionario de la Real Academia Española, 2001). De ahí, que el área incluya el agrupamiento de funciones relacionadas y también las acciones que son propias a los cargos. A continuación, de manera telegráfica, se exponen algunas de 
las áreas más relevantes, recogidas por la Comisión Mixta del Perfil Profesional (2003).

a) Área de atención. La intervención del Trabajo Social se concreta en las acciones con las personas y colectivos que sufren las consecuencias de situaciones carenciales, conflictos y crisis. Su fin es posibilitar el desarrollo humano y autonomía a través de la movilización de sus capacidades y de los recursos.

b) Área de prevención. La intervención profesional se centra en las causas de las dinámicas de marginación, exclusión y desintegración social, con la finalidad de evitar su aparición.

c) Área de promoción y educación. El Trabajo Social se orienta a descubrir y potenciar las capacidades de individuos, grupos y comunidades. También, a generar procesos de participación e implicación, de formación y de organización para ayudar a las personas a la adquisición o incremento de los conocimientos y habilidades sociales que precisan.

d) Área de mediación y arbitraje. A través de este área los trabajadores sociales intervienen para mediar en la resolución de los conflictos que afectan a las familias y grupos sociales, tanto en el interior de sus relaciones, como en su entorno social

e) Areas de planificación y evaluación. La intervención de los trabajadores sociales se desarrolla en el diseño de la planificación y de la evaluación de los planes, programas y proyectos.

f) Areas de gerencia y administración. La intervención profesional se realiza en los procesos de organización, coordinación, dirección y animación del personal hacia la consecución de los objetivos y obtención de resultados de calidad.

g) Area de investigación y docencia. Los trabajadores sociales a través de la investigación tratan de profundizar en el conocimiento de las necesidades y problemas, así como en los procesos de calidad que se relacionan con su propio trabajo profesional. Al mismo tiempo, realizan acciones para que los conocimientos acumulados, las experiencias vividas y las reflexiones teóricas se pongan a disposición de los profesionales, de las personas vinculadas a la acción social y de los futuros trabajadores sociales.

Después de presentadas las características de la intervención en Trabajo Social, vamos a tratar de esbozar los elementos configuradores de la calidad para adentrarnos en la intervención en Trabajo Social desde la calidad integrada en el marco de las organizaciones que nos ocupan. 


\section{II. ¿PARA QUÉ INTERVENIR EN TRABAJO SOCIAL DESDE LA CALIDAD INTEGRADA EN LAS ORGANIZACIONES DE SERVICIOS SOCIALES Y DE BIENESTAR SOCIAL?}

Hemos visto cómo la intervención de los trabajadores sociales está orientada a mejorar la calidad de vida de la población, contribuyendo a superar los problemas y a promover el desarrollo humano. También para contribuir a mejorar la calidad de servicio. Ello supone, centrado en los diversos marcos de las organizaciones de Servicios Sociales de bienestar social, favorecer la autonomía y la participación de los usuarios, con un trato de calidez, al tiempo que mejorar la eficacia y eficiencia de la gestión de servicio. Igualmente avanzar hacia una sociedad con mayor justicia social y solidaridad.

Para lograr estos objetivos, de mejorar la calidad de vida de los usuarios y la calidad de servicio, las organizaciones que nos ocupan, conjuntamente con el personal, están llamadas a promover la mejora de la calidad de vida laboral de los trabajadores que ofrecen sus servicios en las mismas. Estas tres vertientes: calidad de vida, calidad de servicio y calidad de vida laboral, es lo que he denominado calidad integrada (Barranco, 2002a), que entiendo ha de contemplarse en la intervención del Trabajo Social en el marco de las organizaciones que nos ocupan.

\subsection{Consideraciones previas}

Teniendo en cuenta los planteamientos anteriores y abundando en la necesidad de intensificar la intervención en Trabajo Social desde la calidad y, más concretamente, de la calidad integrada, se verifica que la mayor ocupación laboral de los trabajadores sociales se da en estas organizaciones. Así, en Canarias se estima que el $85 \%$ de los trabajadores sociales colegiados desarrollan su actividad en los Servicios Sociales y el $15 \%$ restante lo hacen en otras organizaciones de bienestar social y en otros ámbitos comunitarios, siendo prácticamente inexistente el ejercicio libre de la profesión.

Igualmente, es preciso resaltar que, a partir de la mitad de los años noventa y referidos a España, en estas organizaciones se están implantando diversos modelos estandarizados de calidad. Por otra parte, señalar que en los espacios comunitarios se vienen implantando sistemas de planificación y evaluación de calidad, con la participación de organizaciones públicas, privadas, movimiento civil y del sector mercantil, recogidos en Barranco (2002b).

Además, de esta amplia presencia de los trabajadores sociales en dichas organizaciones y la introducción en las mismas de la cultura de 
la calidad, es básico tener en cuenta la legislación que se va impulsando en materia de calidad. De igual modo, la inclusión de marcos referenciales vinculados a la evaluación de la misma en los diversos planes, programas y proyectos de acción social, a nivel local, nacional, europeo e internacional.

A todo lo anterior, es necesario añadir que la cultura de la calidad está calando en la población y en la sociedad occidental de nuestro milenio. También, los organismos internacionales están intensificando sus recomendaciones para que se implanten sistemas de planificación y evaluación de la calidad en las organizaciones de Servicios Sociales de Bienestar Social, públicas y privadas.

Por estas razones y además porque estoy convencida de que la calidad y, concretamente lo que he denominado calidad integrada, aporta beneficios a las personas, a las organizaciones y a la sociedad, pienso que es preciso que la calidad sea incorporada a la formación de los titulados en Trabajo Social. Al mismo tiempo, considero que la calidad integrada debería de ser incluida en las prácticas y en las investigaciones de los trabajadores sociales con la finalidad de evaluar y mejorar su aplicabilidad. Todo ello, desde los enfoques y estilos propios que caracterizan al Trabajo Social.

En esta dirección el trabajador social Barriga (2000) expresa que la incorporación de la calidad a la práctica profesional es una exigencia ética en las organizaciones de bienestar social y servicios sociales, ya que en estas organizaciones la no calidad conculca sistemáticamente los derechos del cliente. Esta idea, que comparto con el autor, está hoy en la mente de muchos profesionales y en los discursos de los organismos internacionales.

En definitiva, la incorporación de la cultura de la calidad al Trabajo Social es una estrategia profesional que posibilita:

- Legitimar prácticas y modelos de intervención, Guillén y Pérez (2002, p. 213).

- Responder a la demanda social y profesional.

- Aplicar las recomendaciones de las instituciones internacionales y europeas.

- Incorporar los procesos de la evaluación continua a los planes, programas y proyectos sociales.

- Avanzar desde la calidad hacia la investigación sobre la calidad integrada realizada por Barranco (1999), en tres centros de atención a personas con discapacidad. 


\subsection{Concepciones sobre la calidad}

Antes de definir lo que entendemos por calidad, cabe decir que la calidad ha estado siempre presente en la historia de la humanidad y en Trabajo Social, si bien lo novedoso en la época actual es la filosofía, los modelos y la cultura que la sustenta. Modelos y cultura que conectan con los objetivos, valores y principios éticos del Trabajo Social, claramente orientados hacia el desarrollo humano de las personas, la mejora de la gestión y el compromiso por una sociedad más justa con mayores oportunidades para todos y de un medio ecológico saludable y sostenible.

El término calidad es definido por el Diccionario de la Real Academia Española como "la propiedad o conjunto de propiedades inherentes a una cosa, que permiten apreciarla como igual, mejor o peor que las restantes de su especie". También, como "la superioridad o excelencia, con respecto a otras de su especie". Y finalmente, como "calidez en el trato", lo que significa dar calor, irradiar confianza, seguridad, amabilidad, empatía y autenticidad.

En la organización, la calidad es definida como un proceso de mejora continua que se produce dentro de una organización y de ésta con su entorno, con el fin transformar los insumos en productos o servicios. El proceso se inicia con la recepción de la demanda o detección de las necesidades y finaliza con las respuestas dadas para satisfacerlas. Para tal fin la organización precisa de trabajadores, infraestructuras, recursos, formación, protocolos, procesos de coordinación y de un entorno profesional con calidad de vida laboral. Desde el modelo de la Calidad Total aplicado en las organizaciones que nos ocupan y recogido en Setién y Sacanell (2003), se resalta que para conseguir la calidad del proceso se ha de incorporar la evaluación continua, la cual desarrollaremos más adelante.

\subsection{Valores y principios de la calidad}

Los valores y principios que la sustentan, los cuales están orientados hacia la persona, la organización y la sociedad. En esta dirección, hemos visto como en Trabajo Social se parte de los valores de los Derechos Humanos, así como del compromiso ético de hacer el trabajo lo mejor posible para responder a las necesidades, demandas y expectativas de la ciudadanía, de la organización y de la sociedad. Observamos que, en consonancia con estos valores en el Plan Estratégico para el fomento de la calidad de las ONGs 2003-2005, que ha sido liderado por el Instituto de Trabajo Social y Servicios Sociales (INTRESS), se recogen los valores siguientes: 
a) Orientados hacia las personas: defensa de sus derechos, dignidad humana, solidaridad, con la finalidad de satisfacer las necesidades sociales, expectativas y la potenciación de las capacidades humanas.

b) Centrados en la Organización: orientación al cliente, profesionalidad, participación, descentralización de la toma de decisiones, eficacia y gestión global de la misión para mejorar los procesos y resultados de los servicios. Procesos y resultados que están orientados a mejorar la Calidad de Vida de los clientes.

c) Orientados hacia la sociedad: transparencia en la gestión y eficiencia y responsabilidad social para avanzar en una sociedad sostenible.

A partir de este señalamiento global de los valores, en el siguiente cuadro se exponen los principios de la calidad que prevalecen en los Servicios Sociales de la Unión Europea, recogidos en el estudio sobre la evaluación de la calidad realizado por Pallinger (2002).

\begin{tabular}{|c|}
\hline PRINCIPIOS DE MEJORA DE LA CALIDAD DE SERVICIO EN EL MARCO EUROPEO \\
\hline Orientación al usuario que promuevan su implicación y su potenciación. \\
\hline $\begin{array}{l}\text { Participación de los usuarios y profesionales en los sistemas de calidad y en el desarrollo } \\
\text { organizativo. }\end{array}$ \\
\hline $\begin{array}{l}\text { Implantación de sistemas de calidad que sean flexibles, adaptables y relevantes a las } \\
\text { necesidades locales. }\end{array}$ \\
\hline $\begin{array}{l}\text { Mecanismos de prestación del servicio coordinados e integrados que cubran las necesidades de } \\
\text { modo multifacético. }\end{array}$ \\
\hline Continuidad de los servicios y de su fnanciación. \\
\hline $\begin{array}{l}\text { Alianzas con proveedores de servicios, con las entidades que financian, con grupos de interés, } \\
\text { con otros socios. }\end{array}$ \\
\hline $\begin{array}{l}\text { Una cultura de la innovación en las organizaciones de servicios de modo que respondan } \\
\text { flexiblemente a las necesidades y a los requerimientos. }\end{array}$ \\
\hline Sistemas efectivos de evaluación con mecanismos de feedback. \\
\hline $\begin{array}{l}\text { Equipos altamente cualificados que sean capaces de responder a las necesidades de los usuarios } \\
\text { y que desarrollen cambios organizativos que reflejen éstas. }\end{array}$ \\
\hline $\begin{array}{l}\text { Un marco de igualdad / diversidad e igualdad de oportunidades entre mujeres y hombres que } \\
\text { asegure que no se descuidan los roles de las mujeres como cuidadoras, su propio cuidado o sus } \\
\text { necesidades de empleo. }\end{array}$ \\
\hline
\end{tabular}

Fuente: Pallinger (2002).

Además, Pallinger expresa que: "puesto que los servicios sociales públicos están cada vez más abiertos a un marco competitivo, se necesitan urgentemente marcos de políticas sobre la calidad que guíen los procesos de privatización o contratación, de modo que se asegure que lo que orienta la adjudicación de los contratos es la calidad y no el coste" (Pallinger, 2002, p. 13). 
Los principios expuestos y los de la calidad total se ven reflejados en el siguiente dibujo que hemos elaborado, tomando como referente el friso de la calidad (López y Gadea, 1995).

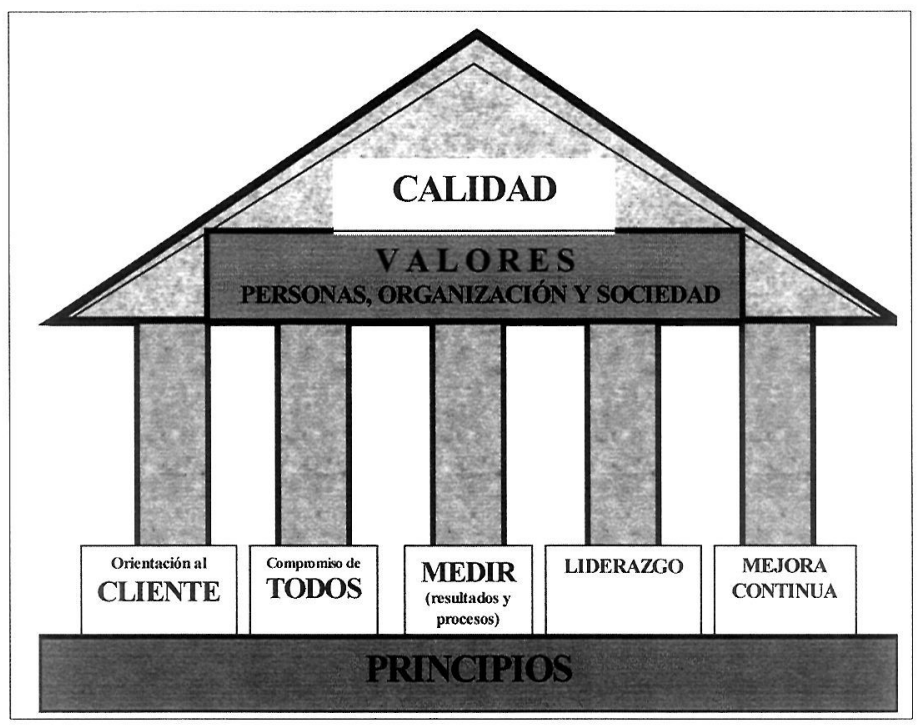

Observamos cómo los valores de la calidad se sustentan en los principios. Valores, que como ya hemos señalado están orientados hacia las personas, la organización y la sociedad. Igualmente de entre los principios expuestos, se remarcan el de participación e implicación de todos, el liderazgo, la gestión por procesos basada en la medición de hechos y datos, la cual se realiza mediante la evaluación continua, que exponemos seguidamente.

\subsection{La evaluación continua}

La evaluación continua es uno de los principios básicos de la calidad. Las misma está plenamente incorporada a la disciplina del Trabajo Social y a la práctica profesional, desde sus comienzos. Si bien, la evaluación continua desde la cultura de la calidad tiene unas caracterísiticas propias tales como los modelos, los procesos y las herramientas que la sustentan, que no están incorporados en el acervo profesional.

Abundando en esta idea, conviene enfatizar que la evaluación continua se convierte en uno de los elementos operativos y fundamentales de la calidad con la participación de los usuarios y de los profesionales. Desde la cultura de la calidad y, más concretamente, el modelo de la Calidad Total, propugna que no se puede hablar de calidad si no se miden los resultados y procesos de la intervención, aplicando para tal fin 
los indicadores que se estimen más idóneos para cada organización y contexto, partiendo de los criterios previamente definidos.

Para Edwards Deming (1981), la evaluación continua conlleva la realización de una serie de ciclos que se han de aplicar de manera sistemática para poder mejorar la gestión de la calidad de servicio. Cada ciclo se estructura en las siguientes fases: planificar, hacer, controlar o verificar y actuar.

La evaluación continua se inicia con la delimitación de los procesos. Para ello se lleva a cabo los siguientes pasos (Colom, 2003):

- Delimitar los objetivos del proceso, sus destinatarios, sus expectativas.

- Describir las actividades, secuencias, recursos y las características de calidad.

- Definir medidas e indicadores.

- Estandarizar procedimientos.

- Revisar y evaluar, midiendo sistemáticamente los puntos fuertes y débiles, así como incorporando las propuestas de mejora.

Las propuestas de mejora deben de someterse a verificación empírica para demostrar su eficacia. Un ejemplo del proceso de autoevaluación continua, utilizada por el Modelo de Gestión de la Calidad de la Fundación Europea, son los formularios de autoevaluación en los cuales se reflejan los criterios y subcriterios establecidos en dicho modelo. En los mismos, el personal de la organización determina los puntos críticos de cada área que han de someterse a evaluación. En cada área, se analizan los puntos fuertes y débiles, finalizando con las propuestas de mejora.

Por último, resaltar que todos los modelos de calidad enfatizan que medir significa comparar con un estándar. No es posible medir sin un referencial previo. Consciente de ello, en las organizaciones de servicios sociales y bienestar social, se están implementado diversos modelos de calidad. Entre los mismos cabe señalar los modelos "EFQM" de la Fundación Europea de Gestión de la Calidad, las Normas "ISO" y la "Norma ONGs con Calidad, que incluyen criterios e indicadores de evaluación.

\subsection{La calidad integrada}

Al profundizar sobre la calidad, observamos que las investigaciones e intervenciones sociales que se vienen realizando han generado un cúmulo importante de estudios y de experiencias sobre la calidad de vida, la calidad de servicio y la calidad de vida laboral. 
Estas tres vertientes de la calidad, tal como hemos indicado, convergen y configuran lo que he denominado la calidad integrada (Barranco, 1999 y 2002a). Así, cada calidad, por separado, tiene identidad propia $y$, al integrarlas en la investigación realizada en tres centros de atención a personas con discapacidad, obtuvimos relaciones directas entre las mismas. En este sentido, se comprueba que en aquellas organizaciones que han obtenido resultados más altos en la calidad de vida, también los han obtenido en la calidad de servicio y en la calidad de vida laboral. El dibujo siguiente ilustra esta idea.

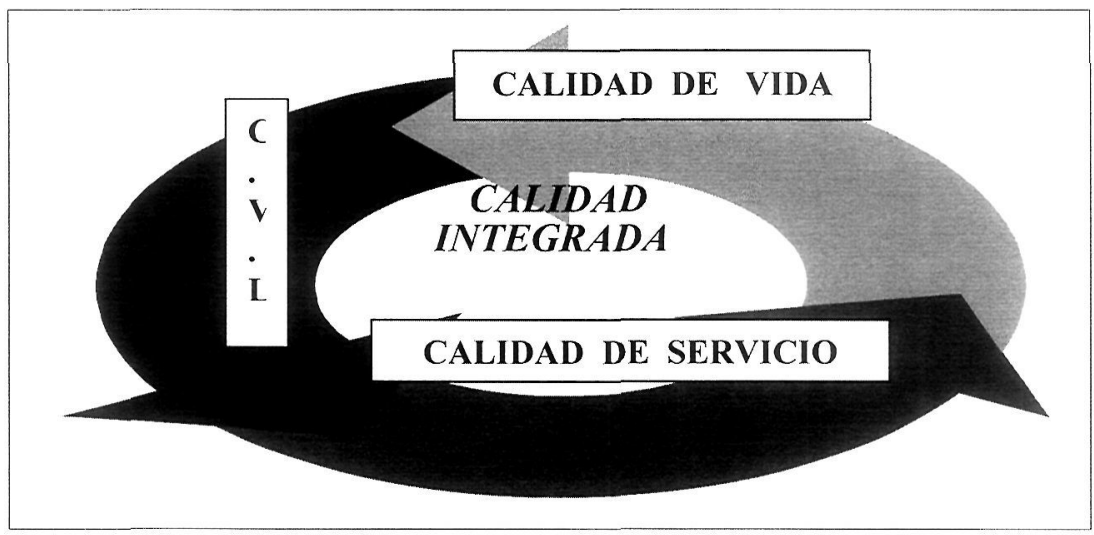

Fuente: Barranco (2002a).

* C.V.L.: calidad de vida laboral.

Aplicado a la intervención en Trabajo Social, como ya se expresó, la calidad integrada incorpora las tres vertientes de calidad siguientes: a) la vertiente "calidad de vida", que orienta la intervención profesional hacia la mejora de la calidad de vida de las personas, grupos o comunidades; b) la vertiente "calidad de servicio", que aborda la eficacia y eficiencia de la intervención profesional en los resultados y procesos de gestión de la organización destinada a obtener los óptimos resultados y el compromiso ético para mejorar la calidad de vida de las personas y la sociedad c) y la vertiente "calidad de vida laboral", que evalúa las condiciones de trabajo en las que se realiza la intervención profesional.

Desde las teorías de la calidad de vida laboral se parte de la premisa de que los trabajadores, para poder contribuir a mejorar la calidad de vida de las personas y la calidad de servicio, tienen que estar motivados y satisfechos con las tareas que realizan, así como con el salario, el ambiente y la salud laboral. En el siguiente dibujo se puede ver representada esta idea de la calidad integrada aplicada a la intervención en Trabajo social desde el marco de las organizaciones de Servicios Sociales de bienestar social. 


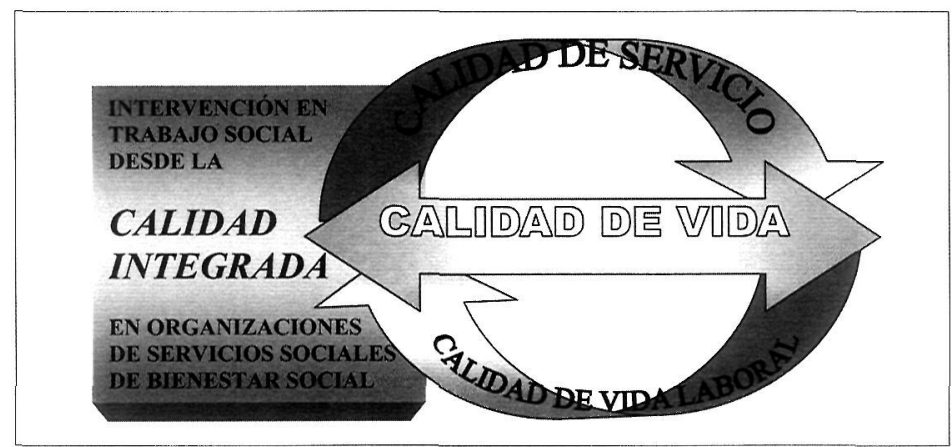

Fuente: elaboración propia.

2.6. Intervención profesional orientada a mejorar la calidad de vida de los usuarios

Uno de los objetivos del Trabajo Social es mejorar la calidad de vida. De ahí, la necesidad de adentrarnos en delimitar qué entendemos por calidad de vida y qué hacen los trabajadores sociales para contribuir a incrementar la calidad de vida de las personas.

La calidad de vida empieza a cobrar carta de naturaleza en las últimas décadas del siglo XX. Parte de la política social y del bienestar social e incorpora la defensa activa del desarrollo humano y del medio ambiente. Ello supone un salto cualitativo con relación al bienestar social al incorporar la vertiente ecológica, la perspectiva del desarrollo humano, tratando de superar los enfoques economicistas y cuantitativistas del Estado de Bienestar, tal como expresa (Alguacil, 2000).

Como concepto, es preciso decir que sobre la calidad de vida no existe una definición consensuada, ya que cada autor parte de dimensiones diferentes (Schaloch y Verdugo, 2003). Una de las definiciones más utilizadas ha sido la de Levi y Anderson (1980). Estos autores definen la calidad de vida como una medida compuesta de bienestar físico, mental y social, tal como la percibe cada persona y grupo. Desde esta perspectiva psicosocial la determinante individual es el grado de coincidencia entre las características de la situación y las expectativas de la persona.

Llegado a este punto es preciso decir que entre los autores hay coincidencia en señalar que la calidad de vida es un constructo multidimensional que incluye las dimensiones personales vinculadas a los espacios de la vida de las personas, así como las dimensiones de los contextos ecológicos en los que se desenvuelven las personas, grupos y comunidades y la valoración que las mismas realizan. 
Como consecuencia, en la evaluación de la calidad de vida intervienen diversas disciplinas, entre las que cabe citar Trabajo Social, Sociología, Economía y Psicología. En su medición se incluyen los aspectos cuantitativos y cualitativos; objetivos y subjetivos; el mundo de las experiencias, vivencias, emociones y cultura.

Entre las dimensiones de calidad de vida vinculadas al mundo de las personas, cabe mencionar la salud (en sus aspectos físicos, psíquicos y sociales), la economía, el trabajo, la vivienda, el medio ambiente, el ocio, los derechos y servicios de bienestar social. Entre las dimensiones del medio social y ambiental, mencionar los ecológicos, los relacionados con el medio social, el círculo familiar, los amigos, los compañeros de trabajo, los sistemas de cultura, de salud, de educación, de empleo, entre otros.

En esta dirección, y sobre los factores ecológicos, la trabajadora social y profesora Cristina de Villalba (2002) incluye los microsistemas o contextos de interacción directa, los mesosistemas o redes sociales (familia, barrio, escuela, trabajo, redes sociales) y el macrosistema o las influencias de los aspectos históricos, socioculturales, políticos, actitudes sociales y regulaciones de una sociedad dada ante los cuidados familiares.

A modo de conclusión y de cara a la intervención del Trabajo Social, conviene enfatizar que la calidad de vida es un concepto multidimensional en el que están presentes tanto los aspectos objetivos como subjetivos de los espacios personales y sociales de las personas, grupos y comunidades, así como los del contexto socioambiental. Por lo tanto, para medir la calidad de vida, se utilizarán tanto los indicadores de salud, empleo, educación, contexto ecológico, servicios, etc., como los aspectos subjetivos, medidos con indicadores basados en las vivencias, percepciones y el grado de satisfacción que tienen las personas sobre sus espacios personales y socio-ambientales.

En cuanto a la cuestión relativa a lo que hacen los trabajadores sociales para contribuir a incrementar la calidad de vida de la ciudadanía, partiendo de las características expuestas de la intervención en Trabajo Social y los planteamientos de la calidad, cabe señalar que estos profesionales intervienen en el diseño de la planificación y de la evaluación de los planes, programas y proyectos de acción social orientados a mejorar la calidad de vida. Implementan y establecen procesos de evaluación continua para valorar en qué medida se ha contribuido a mejorar la calidad de vida en las dimensiones concretas, previamente delimitadas. En esta dirección trabajan y valoran en qué nivel se han incrementado las capacidades, la igualdad de oportunidades, cuáles han 
sido las dificultades personales y los obstáculos del medio social que se han logrado superar o eliminar, entre otros.

En definitiva, incrementar la calidad de vida de la ciudadanía en Trabajo Social implica intervenir con las personas y el medio social desde una perspectiva global que abarca el conjunto de las dimensiones objetivas y subjetivas de sus vidas y las dimensiones del contexto ecológico para:

- Ampliar las capacidades, opciones y oportunidades de las personas, acompañando en sus procesos vitales para ser responsables y ofrecer espacios de participación.

- Facilitar los medios para afrontar los cambios que supongan un obstáculo para el desarrollo humano y la justicia social, promoviendo los recursos necesarios.

- Medir los resultados incluyendo indicadores de calidad de vida correspondientes a las dimensiones de la vida de las personas y del contexto socioambiental a mejorar, tales como salud, empleo, educación, servicios, contexto ecológico, percepciones, vivencias, expectativas, satisfacción.

Finalmente, indicar que para medir la calidad de vida se utilizan escalas validadas como el cuestionario "CUBRECAMI" de FernándezBallesteros, Zamarrón y Macía (1996), aplicado para medir la calidad de vida de las personas mayores. También el cuestionario EUROQOL, utilizado en el ámbito de la salud (Fundación Canaria de Investigación y Salud, 2000). Además, en la investigación realizada por Barranco (1999), así como en la de otros autores, se aplican los cuestionarios "ad hoc" con indicadores cuantitativos y cualitativos, entrevistas abiertas y grupos de discusión.

\subsection{Intervención profesional orientada a mejorar la calidad de ser- vicio: proceso y resultados en la gestión}

La calidad de servicio, entendida como un proceso continuo para mejorar los servicios que se ofrecen a los clientes, está teniendo una gran resonancia en las organizaciones de bienestar social y servicios sociales. También en el resto de las organizaciones sociales y empresas privadas, lo cual constituye uno de los ejes básicos de la gestión de los planes y programas en los diversos ámbitos del marco español, europeo e internacional.

En esta dirección, en el Seminario sobre Calidad de los Servicios Sociales Públicos, organizado por la Fundación Europea para la Mejora de las Condiciones de Vida y de Trabajo (2003), se resaltó que es pre- 
ciso enfocar la intervención social hacia la satisfacción de los clientes y la máxima adecuación de los servicios a las necesidades, a la inclusión social, a la igualdad, a la accesibilidad de servicio y a la valoración del factor humano.

En cuanto a su definición, señalar que el servicio es definido como la organización y personal destinados a cuidar intereses o satisfacer necesidades del público o de alguna entidad oficial o privada (DRAE, 2001). Además, se afirma que el servicio es esencialmente intangible y no da como resultado la propiedad de nada. Concretamente, la calidad de servicio es entendida como:

- El hacer las cosas bien.

- Ofrecer al cliente lo prometido.

- Identificar y satisfacer las expectativas y necesidades de los clientes.

Para evaluar la calidad de servicio, tal como hemos indicado, se aplican los procesos de evaluación continua, partiendo de unos estándares y herramientas cuantitativas que sean fáciles y ágiles de emplear, combinándola con metodologías cualitativas. La evaluación continua se realiza de manera sistemática. Está basada en la medición multidimensional e interdisciplinar, con criterios de eficacia y eficiencia, utilizados tanto en los procesos como en los resultados. Como consecuencia, para mejorar y medir la calidad de servicio, como hemos ido reflejando, es preciso implantar sistemas de calidad utilizando los diversos modelos estandarizados ya reseñados ("EFQM" de la Fundación Europea de Gestión de la Calidad, las Normas "ISO" y la "Norma ONGs con Calidad"). Evaluación que se ha de realizar con la participación de los usuarios y profesionales.

Dada la relevancia del Modelo Europeo de Gestión de Calidad, muy brevemente se harán unas referencias al mismo. Este modelo está orientado a la gestión de la calidad de servicio y se configura como un modelo de autoevaluación interna. Básicamente contempla los criterios ponderados siguientes: liderazgo; planificación y estrategia; gestión de personal; recursos; procesos; satisfacción del cliente; satisfacción del personal; impacto en la sociedad y resultados.

En dicho modelo se considera que la autoevaluación es la clave para la mejora de la gestión de servicio. Autoevaluación entendida como el examen global, sistemático y regular de las actividades y resultados de una organización. La autoevaluación busca ser un instrumento de diagnóstico, basado en:

- Hechos y datos. 
- Un lenguaje y base conceptual común para todo el personal de la organización.

- El análisis de los puntos fuertes y débiles, así como en las propuestas de las áreas a mejorar.

- La valoración y comparación en distintos momentos.

El modo como se realizan las autoevaluaciones atiende a los criterios indicados. Además, a los subcriterios y áreas que deben de ser adaptados a las características de cada organización y unidad con flexibilidad. El resultado del análisis se refleja en un modelo de ficha que contiene la información resultante del proceso de autoevaluación, atendiendo a los aspectos indicados de criterios, subcriterios, áreas, puntos fuertes, puntos débiles y aspectos a mejorar.

En definitiva, la autoevaluación es el elemento clave en el proceso y resultado de la calidad de servicio en el modelo EFQM. Autoevaluación que posibilita identificar los puntos críticos de cada unidad, analizar los puntos fuertes y débiles de la organización y realizar propuestas para mejorar la gestión de servicio.

Especial importancia se concede a la evaluación de las expectativas y percepciones de los clientes. En esta dirección, Zeithaml, Parasuraman y Berry (1993) desarrollaron la escala SERQVUAL, basada en el Modelo de Gestión de la Calidad Percibida. Para estos autores, la calidad de servicio es el grado de discrepancia o coincidencia existente entre las expectativas (valoración de lo que el servicio debería ofrecer) y las percepciones (valoración del servicio recibido).

Este modelo de evaluación de la calidad percibida tiene en cuenta el punto de vista de los clientes internos, los empleados y gerentes, así como el de los clientes externos, los usuarios del servicio, por lo que la escala puede aplicarse a cada uno de los protagonistas de la organización. Dicho instrumento desarrolla cinco dimensiones, cuatro de las cuales son intangibles: fiabilidad, capacidad de respuesta, seguridad y empatía, y una tangible, que hace referencia a las instalaciones físicas, equipamiento y apariencia del personal.

En Canarias, esta escala ha sido aplicada en los centros ocupacionales de personas con discapacidad por Barranco, Cáceres, Cívicos, DíazVilela, Hernández y Puyol (2003), mostrando una fiabilidad alta, en familias, profesionales y gerentes. Por el contrario, la fiabilidad alcanzada por los usuarios obtuvo una menor puntuación. De ahí que el equipo desestime su aplicación para los colectivos de personas con discapacidad de estos centros, en el contexto estudiado de la provincia de Santa Cruz de Tenerife. Entre las posibles causas se hipotetiza el bajo nivel 
cognitivo que dificulta la comprensión y, por tanto, la fiabilidad de las respuestas a cada una de las preguntas recogidas en el SERVQUAL.

Además, se aplican cuestionarios "ad hoc", entrevistas, las cartas servicio y las certeras o catálogos de servicio. Las Cartas de Servicios y la Cartera de Servicios son instrumentos que se empezaron a implantar en diversos países europeos en los Servicios de Bienestar Social. Las Cartas de Servicios tienen como objetivos: a) facilitar a los ciudadanos el ejercicio de sus derechos; b) impulsar iniciativas de mejora dentro de los servicios; c) controlar el grado de cumplimiento de los compromisos contraídos con los ciudadanos; d) incrementar el grado de satisfacción de los ciudadanos respecto a los servicios prestados; e) informar a los ciudadanos sobre los servicios que se prestan y sobre su nivel de calidad (Comunidad de Madrid, 1999; De la Red, 2000; Guillén y Pérez, 2002).

En esta línea, conviene destacar que, de los estudios realizados para evaluar el servicio ofrecido por los trabajadores sociales en Reino Unido, Howe (1999) expone que los resultados de dichos estudios pusieron de manifiesto que: "a los clientes les gusta que los trabajadores sociales se muestren amigables, sencillos y honestos (...) que basen su relación en la empatía, autenticidad y calidez (...) que en la búsqueda de resultados, sean sistemáticos, organizados y claros para decir a los clientes lo que piensan, cómo ven las situaciones, cómo deberían tratarse las cosas, qué están haciendo, dónde están y hacia dónde les gustaría ir (...) buscando la participación y el punto de vista del usuario" (Howe, 1999, p. 29-32).

Finalmente, cabe resaltar que, aunque la cultura de la calidad se está implantando recientemente en las organizaciones de bienestar social y servicios sociales, su origen es anterior, ya que nace en el sector industrial y empieza a tener un gran empuje a partir de los años 50, con los trabajos de los considerados padres de los métodos y herramientas de la calidad (Juran, 1982; Crosby, 1979; Deming, 1981; Feigenbaum, 1984), los cuales han ido elaborando e implementando el diseño y las herramientas para medir la calidad. Sin duda, estos autores sentaron las bases de los enfoques actuales de la calidad, y de sus planteamientos parten la mayoría de los modelos referenciados.

\subsection{La calidad de vida laboral: mejorar las condiciones laborales de los profesionales}

La calidad de vida laboral es entendida como una filosofia enfocada a humanizar el trabajo, teniendo como meta mejorar las condiciones 
laborales, incrementar la satisfacción laboral de los trabajadores, así como mejorar la eficacia y eficiencia organizacional.

En esta línea, se parte de la premisa que, para poder ayudar a otras personas y ofrecer un servicio con calidad y eficacia, es preciso que los trabajadores que prestan los servicios tengan unas adecuadas condiciones laborales. Ello significa tener satisfacción laboral, apoyo social laboral y evitar el burnout. Debido a las amplias repercusiones negativas que tiene el síndrome del burnout, es fundamentar evitar su aparición, tal como recoge Barranco (2004). Asimismo, se ha de potenciar la motivación laboral, resaltada en estos servicios por Gutiérrez Resa (2002). Algunos de estos aspectos están recogidos en la Ley 16/2003 de 28 de mayo, de "Cohesión y Calidad del Sistema Nacional de Salud", publicada en España, que incluye la formación y la satisfacción laboral.

Para medir la Calidad de Vida Laboral en los Servicios Sociales se cuenta con las escalas adaptadas por Gil-Monte (1994), aplicadas por este autor y por Barranco (1999) en diversos contextos hospitalarios y Centros de Atención de Personas con Discapacidad, respectivamente. Dichas escalas recogidas en la publicación de Barranco (2002a) son: a) Apoyo Social Laboral, "Organizational Stress Questionarie" (OSQ) (Caplan, Cobb, French, Van Harrison y Pinneau, 1975); b) Satisfacción laboral, "Cuestionario de Satisfacción S20/23" de Meliá y Peiró, (1989) y c) El burnout, "Maslach Burnout Inventory" (MBI) de Maslach y Jackson, (1986).

Para terminar este apartado, destacar que la calidad de vida laboral ha de ser un referente para aquellas organizaciones sociales que se preocupen por aplicar las directrices internacionales, europeas y españolas en materia de salud laboral. Desde estos foros, se preconiza, que para incrementar la calidad de vida de la población y la calidad de servicio, es preciso fomentar la calidad de vida laboral, por las consecuencias positivas que la misma tiene para los trabajadores, los usuarios de los servicios, la organización y para la sociedad.

\section{REFLEXIONES FINALES}

En base a los planteamientos expuestos a lo largo de este artículo, pienso que la intervención en Trabajo Social, en el ámbito de las organizaciones de Servicios Sociales de bienestar social, es preciso encararla desde la calidad integrada. Esta propuesta, tal como se ha ido señalando, se fundamenta en los resultados de los estudios realizados y en la aplicación de la ética profesional del Trabajo Social. 
Así, en los estudios efectuados en tres centros de atención a personas con discapacidad se evidencia la relación existente entre las tres vertientes: calidad de vida, calidad de servicio y calidad de vida laboral. De igual manera, hemos visto cómo el objeto, los objetivos y la ética del Trabajo Social están orientados hacia las personas en interacción con el medio social para promover el desarrollo humano y mejorar la calidad de vida del conjunto de la sociedad. De ahí, que la calidad integrada puede contribuir a concretar estrategias basadas en la filosofía, los valores y los principios propugnados desde el Trabajo Social que contribuyan a:

- Mejorar la calidad de vida de las personas, grupos y comunidades. La finalidad es poner a las personas en el centro de la intervención profesional para satisfacer sus necesidades, potenciar sus capacidades, contribuir a eliminar los obstáculos personales y los del medio social, para avanzar en el desarrollo humano, potenciando la igualdad de oportunidades y la justicia social de toda la ciudadanía.

- Mejorar la calidad de servicio. Ello significa incrementar la eficacia y la eficiencia de la gestión en la organización, desde los estilos propios de los trabajadores sociales. Estilos que se caracterizan por la responsabilidad profesional con las personas, las organizaciones y la sociedad. Además, por buscar maximizar los recursos; establecer procesos de comunicación basada en la calidez en el trato, la atención integral, la empatía y la autenticidad.

- Mejorar la calidad de vida laboral. La finalidad es lograr que las organizaciones tengan como misión potenciar una sociedad saludable, justa y solidaria. Ello implica intensificar los esfuerzos para que los trabajadores estén satisfechos, tengan apoyo laboral y evitar el burnout o síndrome de estar quemado.

Estas son algunas de las razones por las que considero que incorporar la calidad integrada en la intervención del Trabajo Social es útil socialmente. Resaltar que esta perspectiva de la calidad integrada puede incluirse en los modelos de calidad estandarizados y referenciados, equilibrando, la enfatizada y desarrollada "gestión económica" con la vertiente social de "desarrollo humano y de la calidad de vida".

En definitiva, la calidad integrada en las organizaciones de Servicios Sociales de bienestar social puede contribuir a potenciar en las mismas la imagen poliédrica de la igualdad de oportunidades para toda la ciudadanía, la transparencia, la responsabilidad social, la orientación ecológica, la eficacia, la eficiencia y la calidez humana. 


\section{BIBLIOGRAFÍA}

ALGUACIL, J. (2000). La calidad de vida y práxis urbana. Madrid: CIS.

BARRANCO, C. (1999). Estudio de los CAMPs de Tenerife. Tesis doctoral. La Laguna: Universidad de la Laguna, Departamento de Didáctica y Ciencias del Comportamiento.

BARRANCO, C. (2002a). La Calidad percibida en Centros de Atención a Personas con Discapacidad Psiquica de Tenerife. Un enfoque integrado de la Calidad de Vida, Calidad de Servicio y Calidad de Vida Laboral. Tenerife: Cabildo de Tenerife.

BARRANCO, C. (2002b). Calidad de vida y Trabajo Social. Planteamientos teóricos y experiencias en organizaciones de bienestar social y en comunidades. AGATHOS, 4, 4-21.

BARRANCO, C. (2004) Los profesionales de ayuda y el burnout. Trabajo Social y Salud, 47, 27-37.

BARRANCO, C.; CÁCERES, C.; CÍVICOS, A.; DÍAZ VILELA, F. L.; HERNÁNDEZ HERNÁNDEZ, M. y PUYOL, B. (2003). Informe de la Evaluación de la Calidad Servicio de los Centros Ocupacionales de la Provincia de Santa Cruz Tenerife. Tenerife: Universidad de La Laguna. Departamento de Trabajo Social y Servicios Sociales (sin editar).

BARRIGA, L. A. (2000). Calidad en los servicios sociales reflexiones sobre la calidez total. Revista de Servicios Sociales y Politica Social, 49, 31-62.

CAPLAN, R. D.; COBB, S; FRENCH, J. R. P.; VAN HARRISON, R. y PINNEAUA, S. R. (1975): Job demands and worker health. En Gil-Monte, P (1994): El sindrome de Burnout: un modelo multicausal de antecedentes y consecuentes en profesionales de enfermeria. La Laguna, Tesis Doctoral, Universidad de La Laguna.

COLOM, D. (2003). El Trabajo Social Hospitalario en transformación: hacia nuevos retos y la mejora de las competencias. Servicios Sociales y Política Social, 64, 75-103.

COMISIÓN MIXTA DEL PERFIL PROFESIONAL (2003). Perfil Profesional del Trabajador Social Siglo XXI. Madrid: documento interno.

COMUNIDAD DE MADRID (1999). Satisfacción de los ciudadanos y ciudadanas. Madrid: Dirección General de Calidad de los Servicios.

CONSEJO ESTATAL DE ONG DE ACCIÓN SOCIAL (2003). Proyecto de plan estratégico para el fomento de la calidad de las organizaciones no gubernamentales 2003-2005. ONG con calidad. Recuperado el 14 de Abril de 2003, de htpp: //ww.ongconcalidad.org/actualidad.htm

CONSEJO GENERAL DE COLEGIOS OFICIALES DE DIPLOMADOS EN TRABAJO SOCIAL Y ASISTENTES SOCIALES (1999). La ética del trabajo social principios y criterios. Recuperado el 14 de abril de 1999, de htpp://www.cgtrabajosocial.es/

CONSEJO GENERAL DE COLEGIOS OFICIALES DE DIPLOMADOS EN TRABAJO SOCIAL Y ASISTENTES Sociales de España, (2003). Perfil profesional trabajador social siglo $X X I$ (borrador). Madrid: documento inédito.

CROSBY, P.B. (1979): Quality is Free. Nueva York, MacGraw-Hill.

DEMING, E.W. (1981): Management and Stadistical Techniques for Quality and Productivity. New York. New York University (School Business).

FERNÁNDEZ-BALLESTEROS, R.; ZAMARRÓN, M.D. y MACÍA, A. (1996). Calidad de vida en la vejez en distintos contextos. Madrid: INSERSO.

FEIGENBAUM, A.V. (1984): «Product quality: how to get it; how to keep it». Chief Executive, $\mathrm{n}^{\circ} 27$, págs. 20-23.

FUNDACIÓN CANARIA DE INVESTIGACIÓN Y SALUD (2000). Cuestionario EuroQol. Tenerife: FUNCIS.

FUNDACIÓN EUROPEA PARA LA MEJORA DE LAS CONDICIONES DE VIDA Y TRABAJO (2002). Seminario: La calidad de los servicios sociales públicos. Resultados y conclu- 
siones. Irlanda: Fundación Europea para la Mejora de las Condiciones de Vida y de Trabajo. Recuperado el 15 de mayo de 2003, de http://www.eurofound.eu.int.

GIL-MONTE, P. (1994): Tesis Doctoral. La Laguna: Universidad de La Laguna.

GUILLÉN, E. y PÉREZ, D. (2002). Procesos de mejora: calidad, decisiones e innovación. En T. Fernández y A. Ares (Coords.). Servicios Sociales: Dirección, gestión y Planificación. Madrid: Alianza.

GUTIÉRREZ RESA, A. (2002). Motivación en los Servicios Sociales. En T. Fernández y A. Ares (Coords.). Servicios Sociales: Dirección, gestión y Planificación. Madrid: Alianza.

HOWE, D. (1999). Dando sentido a la práctica. Una introducción a la teoria del Trabajo Social. Granada: Maristan. (Orig. 1992).

JURAN, J.M. (1982): Upper Managemenet and Quality. Nueva York, Juran Institute

LÓPEZ, J. y GADEA, A. (1995). Servir al ciudadano. Gestión de la Calidad en la Administración Pública. Barcelona: Ediciones Gestión 2000.

LEVI, L. y ANDERSON, L. (1980): La tensión psicosocial. Población, ambiente y calidad de vida. México D. F., El Manual Moderno.

MASLACH, C. y JACKSON, S.E. (1986). Maslach Burnout Inventory (2nd. ed.). Palo Alto, Consulting Psychologists Press. En P. Gil-Monte. (1994). Sindrome de burnout: un modelo multicausal de antecedentes y consecuentes en profesionales de enfermería. Tesis doctoral, Universidad de La Laguna.

MARTÍNEZ ROMÁN, M.A. (2003). Consideraciones generales: relaciones del Trabajo Social con el bienestar social, Estado de bienestar, política social, servicios sociales, diferenciación de conceptos. En T. Fernández y C. Alemán (coords.). Introducción al Trabajo Social. Madrid: Alianza.

MELIÁ, J.L. y PEiRÓ, J.M. (1989). La medida de la satisfacción laboral en contextos organizacionales: el cuestionario de satisfacción S20/23. Psicologema, 3-5, 59-74.

PALLINGER, J. (2002). Disability and Quality of Services: Irish and European Perspectives. Recuperado el 19 de agosto de 2002, de http:/www.policyinstitute.tec.ie

Schalock, R. y VERDUGO, M.A. (2003). Calidad de vida. Manual para profesionales de la educación, salud y servicios sociales. Madrid: Alianza.

RED, N. DE LA, (1996). Epistemología y Metodología de Trabajo Social. En I Congreso Estatal de Escuelas Universitarias de Trabajo Social. Valencia: Apuntes de la ponencia.

RED, N. DE LA, (2000). La Metodología en el Trabajo Social desde el marco de las ciencias sociales. CABS. Cuadernos Andaluces de Bienestar Social, 8, 9-32.

RUEDA, D. (2000). Marco operativo de la Intervención Integral. Intervención Psicosocial, I (9), $153-178$

REID, W. (2003). El conocimiento aplicado a la práctica directa del trabajo social: un análisis de tendencias. RTS. Revista de Treball Social, 169, 7-51.

SETIÉN, M. y SACANELL, E. (coords.) (2003). La calidad en los Servicios Sociales: concepios y experiencias. Valencia: Tirant Lo Blanch.

VILLALBA, C. (2002). Abuelas cuidadoras. Valencia: Tirant to Blanch.

ZEITHAML, V.; PARASURAMAN, A. y BERRY, L. (1993). Calidad tolal en la gestión de servicios. Cómo lograr el equilibrio entre las percepciones y las expectativas de los consumidores. Madrid: Ediciones Díaz Santos. (Orig. 1990). 